\title{
High-Dose Vitamin C Administration Inhibits the Invasion and Proliferation of Melanoma Cells in Mice Ovary
}

\author{
Kentaro Nakanishi, ${ }^{a, b}$ Keiichi Hiramoto, ${ }^{a}$ Eisuke F Sato, ${ }^{a}$ and Kazuya Ooi ${ }^{*, a}$ \\ ${ }^{a}$ Department of Pharmaceutical Science, Suzuka University of Medical Science; Suzuka, Mie 513-8670, Japan: and \\ ${ }^{b}$ Medical Nakanishi Co., Ltd.; 738-2 Idocho, Kumano, Mie 519-4324, Japan. \\ Received August 8, 2020; accepted October 1, 2020
}

Several studies have been conducted to explore the anticancer effects of vitamin C (VC). However, the effect of high-dose VC administration on melanoma is still unknown. Therefore, in this study, we investigated the effects of high-dose $\mathrm{VC}(4 \mathrm{~g} / \mathrm{kg})$ on the invasion and proliferation of melanoma cells in various organs of mice. B16 melanoma cells $\left(1 \times 10^{6}\right.$ cells $\left./ 100 \mu \mathrm{L}\right)$ were intravenously injected into the tails of female mice, and VC solution $(4 \mathrm{~g} / \mathrm{kg})$ was orally administered once a day for $14 \mathrm{~d}$. On the 15 th day, samples from the liver, lungs, jejunum, and ovaries were collected and analyzed for invasion and proliferation of melanoma cells. Oral VC administration decreased the number of dihydroxyphenylalanine (DOPA)-positive cells and gp100positive melanoma cells in the ovaries and suppressed the invasion and proliferation of melanoma. Compared to melanoma-administered mice, macrophage inflammatory protein-2 levels and number of neutrophils were increased in the VC + melanoma-administered mice. Furthermore, the concentrations of VC, iron, and hydrogen peroxide, and the number of terminal deoxynucleotidyl transferase mediated deoxyuridine triphosphate nick end labeling (TUNEL)-positive cells were significantly increased in the ovaries of VC + melanomaadministered mice compared to those of melanoma-administered mice. These results suggest that VC can reduce the invasion and proliferation of melanoma cells in the ovaries, and neutrophils in the ovaries play an important role in achieving this melanoma-suppressive effect.

Key words vitamin C; melanoma; ovary; neutrophil

\section{INTRODUCTION}

Melanoma is a malignant tumor of melanocyte cells, and it is thought to be caused by carcinogenesis of melanocytes in the basal layer of the epidermis. ${ }^{1)}$ Although most melanomas occur in the skin, they have also been found in the mouth, intestines, and eye, owing to the distribution of melanocytes. ${ }^{2)}$ Though the morbidity of melanoma is 1 to 2 people per 100000 in Japan, the number has been increasing in recent years. ${ }^{3)}$ The exact cause of melanoma is still unknown, but it is assumed to be triggered by genetic predisposition and environmental factors. ${ }^{4}$ For example, the melanoma incidence in Caucasians is several times higher than Hispanics and blacks, and the incidence in Whites living in areas of high UV intensity is higher than those living in areas of low UV intensity, suggesting the involvement of UV radiation in the etiology of melanoma. ${ }^{5)}$ In Japan, the majority of melanomas arise at sites which are less exposed to UV radiation, such as the trunk and proximal limbs. ${ }^{6}$ In rare cases, melanoma is also found in the ovaries. ${ }^{7-9)}$

Standard treatments for melanoma include surgical resection, chemotherapy, radiation therapy, biological therapy, and targeted therapy ${ }^{10)}$ However, the prognosis of malignant melanoma in the gynecological field is poor and depends on how well it can be resected by surgery. ${ }^{11)}$

Furthermore, vitamin C (VC) is a widely used general purpose medication and OTC drug. ${ }^{12)} \mathrm{VC}$ is involved in collagen formation, prevention of anemia, and inhibition of melanin production. In addition, $\mathrm{VC}$ is also an antioxidant which removes reactive oxygen species (ROS), however, in the presence of ferric iron, $\mathrm{VC}$ can also act as a pro-oxidant, gen- erating ROS such as hydroxyl radical and hydrogen peroxide $\left(\mathrm{H}_{2} \mathrm{O}_{2}\right){ }^{13,14)}$ In recent years, the use of VC infusion therapy in cancer treatment has attracted attention, as it does not exhibit adverse drug reactions. This treatment is already undergoing clinical trials in the United States and being widely tested as a personalized treatment modality in Japan, the cost of which is borne by the patients themselves. ${ }^{15,16)} \mathrm{A}$ treatment combining high-dose $\mathrm{VC}$ and anticancer drugs uses the mechanism of action of pro-oxidants. Compared to normal cells, cancer cells have lower levels of antioxidant enzymes such as catalase, therefore, ROS generated by VC are thought to act selectively on cancer cells and cause apoptosis. ${ }^{17)}$ However, the effect of high-dose $\mathrm{VC}$ on the invasion and proliferation of melanoma cells is unknown.

Thus, in this study, we investigated the effect of high-dose $\mathrm{VC}$ on the invasion and proliferation of melanoma cells using C57BL/6J and gp91 phox, a reduced nicotinamide adenine dinucleotide phosphate (NADPH) oxidase subunit, knockout (gp91 $1^{\text {phox-l-}}$ ) mice. In particular, we examined the organspecific action of VC.

\section{MATERIALS AND METHODS}

Animal Experiments Female C57BL/6J mice (7 weeks old; Japan SLC, Hamamatsu, Shizuoka, Japan) and gp91 phox-1mice (Jackson Laboratories, Bar Harbor, ME, U.S.A.) were reared at a constant temperature of $23 \pm 1{ }^{\circ} \mathrm{C}$ with $50 \pm 10 \%$ relative humidity under a 12 -h light/12-h dark cycle. At 8 weeks of age, mice were randomly assigned to four groups ( $n=6$ per group): control, high-dose VC, melanoma, and $\mathrm{VC}+$ melanoma. B16 melanoma cells lines (Cell Resource 

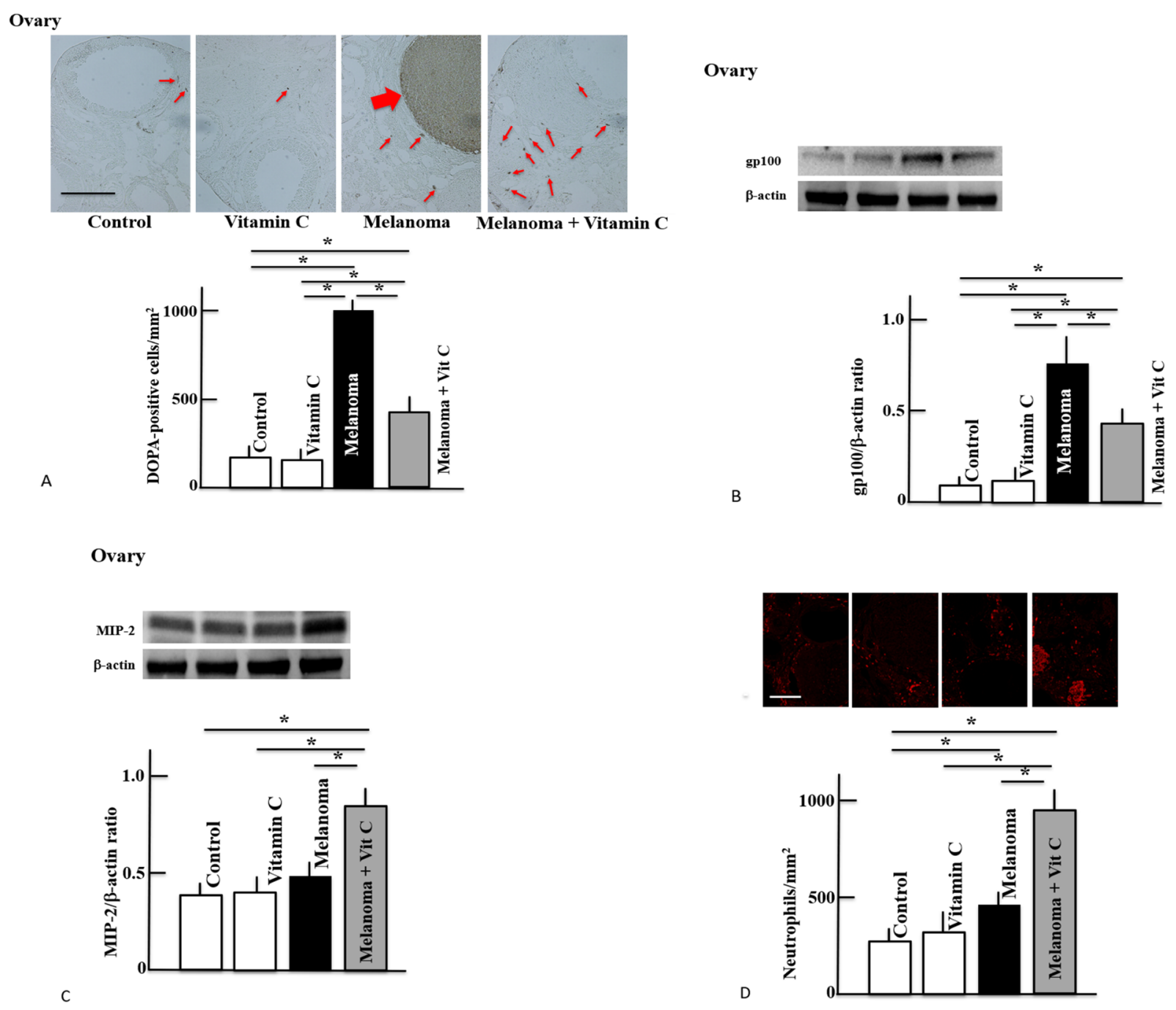

C

Fig. 1. Effect of High-Dose Vitamin C on the Invasion and Proliferation of Melanoma Cells in the Ovary

The number of DOPA-positive cells (indicated by arrows indicate in the images) (A), the expression of gp100-positive melanoma cells (B), MIP-2 levels (C), and the number of neutrophils (D) in the experimental and control groups. The date are represented as mean \pm standard deviation (S.D.). Tukey's post-hoc test was used to compare the differences between the groups. ${ }^{*} p<0.05$. Scale bar $=100 \mu \mathrm{m}$. Abbreviation: Vit C, vitamin C. (Color figure can be accessed in the online version.)

Center for Biomedical Research, Tohoku University, Sendai, Japan) were used at passages 5 to 15 , and the melanoma cells were cultivated as previously described ${ }^{18)}$ and intravenously injected $\left(1 \times 10^{6}\right.$ cells $\left./ 100 \mu \mathrm{L}\right)$ into the tail of the mice to induce melanoma. This study was conducted in strict accordance with the recommendations of the Guide for the Care and Use of Laboratory Animals of Suzuka University of Medical Science (Approval No. 66). All surgeries were performed under pentobarbital anesthesia and every effort was made to minimize animal suffering.

VC Treatment To determine the effective VC dose, we examined the effect of $1,4,8$, or $12 \mathrm{~g} / \mathrm{kg}$ of VC (Fuso Pharmaceutical Industries Ltd., Osaka, Japan) solution in distilled water, and found high and equivalent effectiveness at $4 \mathrm{~g} / \mathrm{kg}$ and higher doses (data not shown). Thus, mice in experimental groups were orally administered approximately $4 \mathrm{~g} / \mathrm{kg}$ of $\mathrm{VC}$ in distilled water for $14 \mathrm{~d}$. The control animals were administered distilled water. ${ }^{19)}$

Preparation and Staining of Tissue Specimens On the 15th day, we extracted lung, liver, jejunum, and ovary samples under anesthesia. The tissue samples were fixed with phosphate buffered saline (PBS) containing 4\% paraformaldehyde
(Wako Pure Chemical Industries, Ltd., Osaka, Japan) and then embedded in Tissue-Tek OCT Compound (Sakura Finetek, Tokyo, Japan) to cut into $5 \mu \mathrm{m}$ thick sections. The dihydroxyphenylalanine (DOPA)-positive cells, which are markers of melanocytes rich in tyrosinase in each tissue, were stained as described previously. ${ }^{20)}$ In addition, the sections were immunohistochemically stained for neutrophils ${ }^{21)}$ using antibodies against neutrophil-specific antigen Ly-6C (100:1; Bio-Rad laboratories Inc., Hercules, CA, U.S.A.). Briefly, the sections were incubated with antibodies overnight at $4{ }^{\circ} \mathrm{C}$ washed with PBS, and incubated with fluorescein isothiocyanate (FITC)labeled anti-rabbit antibody $(1: 30$; Dako Cytomation, Glostrup, Denmark) for $2 \mathrm{~h}$ at $25^{\circ} \mathrm{C}$. Further apoptosis was analyzed using the in situ apoptosis detection kit (TaKaRa, Shiga, Japan). The numbers of DOPA-positive cells, neutrophils, and terminal deoxynucleotidyl transferase mediated deoxyuridine triphosphate nick end labeling (TUNEL)-positive cells were determined using an analysis software Image J v20 (National Institutes of Health, Bethesda, MD, U.S.A.). Five observation fields $\left(1 \mathrm{~mm}^{2}\right)$ were randomly analyzed from the observed images and the average number of cells per field was determined using the image processing software Image J v20. 

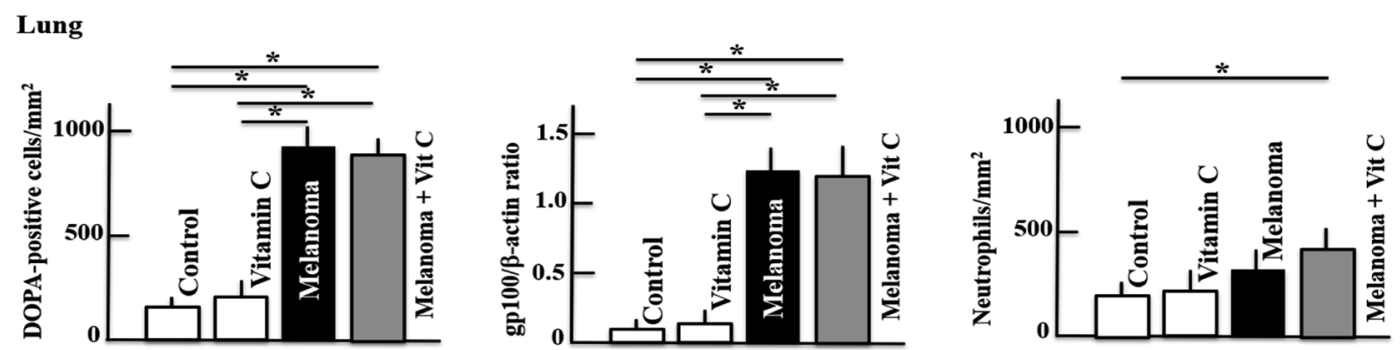

Fig. 2. Effect of High-Dose Vitamin C on the Invasion and Proliferation of Melanoma Cells in the Lungs

The number of DOPA-positive cells, gp100-positive melanoma cells, and neutrophils in the experimental and control groups. The data are represented as mean \pm S.D. Tukey's post-hoc test was used to compare the differences between the groups. ${ }^{*} p<0.05$. Abbreviation: Vit C, vitamin C.
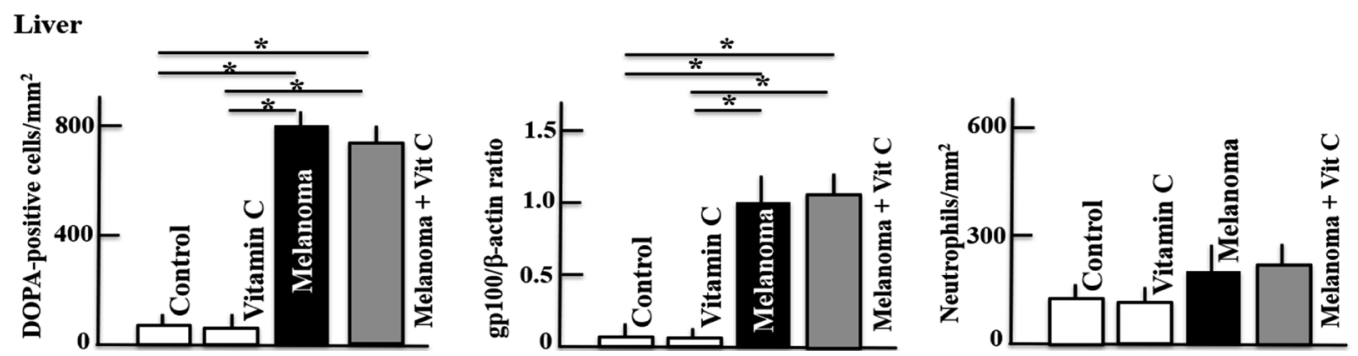

Fig. 3. Effect of High-Dose Vitamin $\mathrm{C}$ on the Invasion and Proliferation of Melanoma Cells in the Liver

The number of DOPA-positive cells, gp100-positive melanoma cells, and neutrophils in the experimental and control groups. The data are represented as mean \pm S.D. Tukey's post-hoc test was used to compare the differences between the groups. ${ }^{*} p<0.05$. Abbreviation: Vit $\mathrm{C}$, vitamin C.
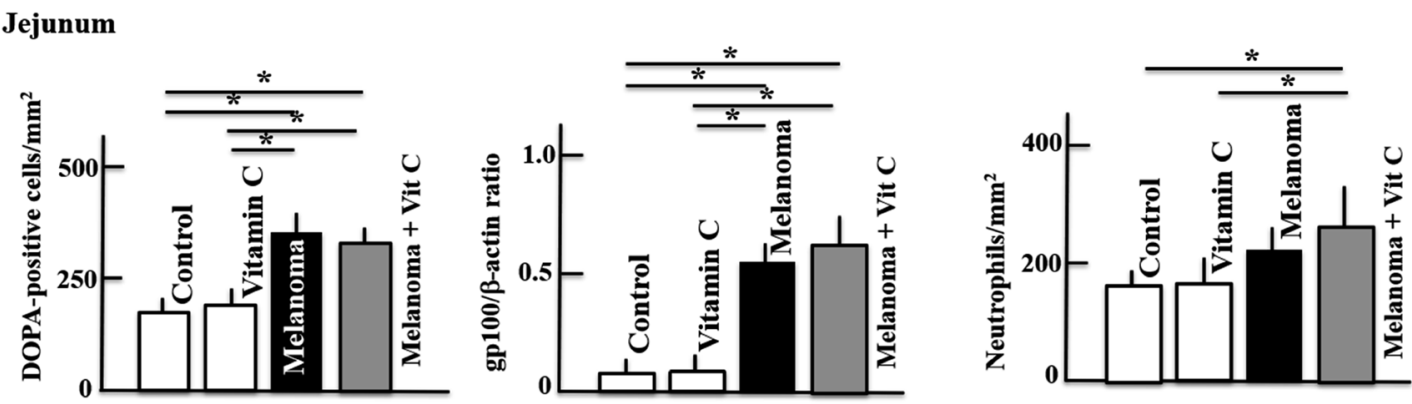

Fig. 4. Effect of High-Dose Vitamin C on the Invasion and Proliferation of Melanoma Cells in the Jejunum

The number of DOPA-positive cells, gp100-positive melanoma cells, and neutrophils in the experimental and control groups. The data are represented as mean \pm S.D. Tukey's post-hoc test was used to compare the differences between the groups. ${ }^{*} p<0.05$. Abbreviation: Vit $\mathrm{C}$, vitamin $\mathrm{C}$.

Western Blotting The ovary samples were homogenized with lysis buffer (Kurabo, Osaka, Japan) and centrifuged at $8000 \times \boldsymbol{g}$ for $10 \mathrm{~min}$. Then, the supernatant was separated and stored at $-80^{\circ} \mathrm{C}$ until further analysis. Western blotting was performed as previously described. ${ }^{22}$ Briefly, the samples were separated by electrophoresis and the membranes were incubated with primary antibodies gp100, a melanoma marker, (1:100; Abcam, Cambridge, U.K.) macrophage inflammatory protein-2 (MIP-2) (1:100; R\&D Systems, Minneapolis, MN, U.S.A.), and $\beta$-actin (1:5000; Sigma-Aldrich Corp., St. Louis, MO, U.S.A.) at $25^{\circ} \mathrm{C}$ for $1 \mathrm{~h}$. Immune complexes on the membranes were visualized with horseradish peroxidase-conjugated secondary antibody (1 : 1000; Novex, Frederick, MD, U.S.A.) and detected with ImmunoStar Zeta regent (Wako Pure Chemical Industries, Ltd.). The images were acquired using the Multi-Gauge Software Program ver. 3.0 (FUJIFILM, Greenwood, SC, U.S.A.).

Neutrophil Separation Method from Ovary Homogenate Neutrophils in the supernatant of the ovary homogenate were separated using an isolation kit (Neutrophil isolation kit;
Cayman, AnnArbor, MI, U.S.A.), and then the number of neutrophils in $1 \mathrm{~mm}^{2}$ was calculated using a hemocytometer (Sunlead Glass Corp., Koshigaya, Japan).

VC Concentration in the Ovaries and Separated Neutrophils The VC concentration in the ovary supernatant, and separated neutrophils was measured using the VC measurement kit (Ascorbate assay kit; Cayman). The VC concentration in neutrophils was calculated as the concentration per fixed number of cells, based on the number of cells calculated as described above.

Measurement of $\mathrm{H}_{2} \mathrm{O}_{2}$ and Iron in Ovary The concentrations of $\mathrm{H}_{2} \mathrm{O}_{2}$ and iron in the ovaries were measured using the OxiSelect ${ }^{\mathrm{TM}}$ STA-347 in vivo ROS/RNS assay kit (Cell Biolabs, Inc., San Diego, CA, U.S.A.) and metalloassay kit (Metallogenics, Chiba, Japan), respectively in accordance with the manufacturer's instructions.

Statistical Analysis The results were analyzed using the Microsoft Excel 2010 software (Microsoft Corp., Redmond, WA, U.S.A.). Differences between groups were evaluated using one-way ANOVA followed by Tukey's post-hoc test in 
the Statistical Package for the Social Sciences (SPSS) v20.0 software (SPSS, Inc., Chicago, IL, U.S.A.). Data are presented as mean \pm standard deviation (S.D.). The results were considered significant at $p<0.05$.

\section{RESULTS}

Effect of High-Dose VC Administration on the Number of Neutrophils and the Invasion and Proliferation of Melanoma Cells in the Ovary In the ovaries of melanomaadministered mice, the numbers of DOPA-positive cells and gp100-positive melanoma cells were increased (Figs. 1A, B). However, in the $\mathrm{VC}+$ melanoma-administered mice group, the numbers of both cell types were significantly decreased $(p<0.05)$ compared with those in melanoma-administered mice (Figs. 1A, B). Further, the number of neutrophils and MIP-2 levels were highest in the ovaries of $\mathrm{VC}+$ melanomaadministered mice (Figs. 1C, D).

Effect of High-Dose VC Administration on the Number

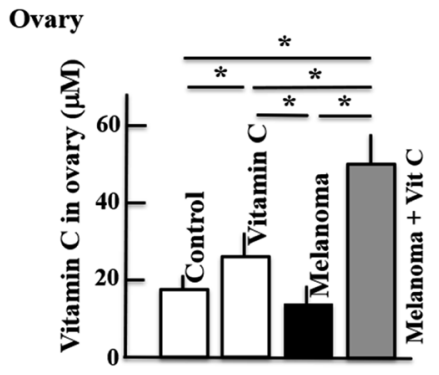

A

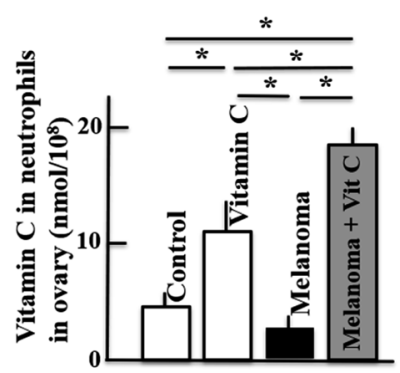

B
Fig. 5. Effects of High-Dose Vitamin C Administration on the VC Concentration in the Ovary Homogenate (A) and in the Neutrophils (B) in the Ovary Samples of the Experimental and Control Groups

The data are represented as mean \pm S.D. Tukey's post-hoc test was used to compare the differences between the groups. ${ }^{*} p<0.05$. Abbreviation: Vit C, vitamin C. of Neutrophils and the Invasion and Proliferation of Melanoma Cells in the Lungs, Liver, and Jejunum The numbers of DOPA-positive cells, gp100-positive melanoma cells, and neutrophils were significantly increased $(p<0.05)$ in the lung, liver, and jejunum in melanoma-administered mice, but were not significantly different when compared to those of $\mathrm{VC}+$ melanoma-administered mice (Figs. 2-4) this, further analysis investigating the effects of high-dose VC administration focused on ovary.

VC Concentration in the Ovary Homogenate and Separated Neutrophils VC concentration in the ovary homogenate and separated neutrophils was significantly lower $(p<0.05)$ in melanoma-administered mice than that in $\mathrm{VC}$ administered mice, but was highest in $\mathrm{VC}+$ melanoma-administered mice (Figs. 5A, B).

Effect of High-Dose VC Administration on the Concentrations of $\mathrm{H}_{2} \mathrm{O}_{2}$ and Iron, and TUNEL-Positive Cells in the Ovaries Next, we measured the concentration of $\mathrm{H}_{2} \mathrm{O}_{2}$ and iron in the ovaries. which is closely related to $\mathrm{VC}$ administration.

The iron and $\mathrm{H}_{2} \mathrm{O}_{2}$ concentration, and the number of TUNEL-positive cells in the ovaries were significantly increased $(p<0.05)$ in $\mathrm{VC}+$ melanoma-administered mice than those in melanoma-administered mice (Figs. 6A-C).

Effect of High-Dose VC Administration on the Number of Neutrophils and the Invasion and Proliferation of Melanoma Cells in the Ovaries of gp91 ${ }^{\text {phox-l- }}$ Mice In the ovaries of gp91 phox-/- mice, the numbers of DOPA-positive cells and gp100- positive melanoma cells increased in the melanoma group and $\mathrm{VC}+$ melanoma group, but did not vary greatly between both these groups (Figs. 7A, B). Similary the number of neutrophils was not significantly different between the melanoma group and the $\mathrm{VC}+$ melanoma-administered group (Fig. 7C).
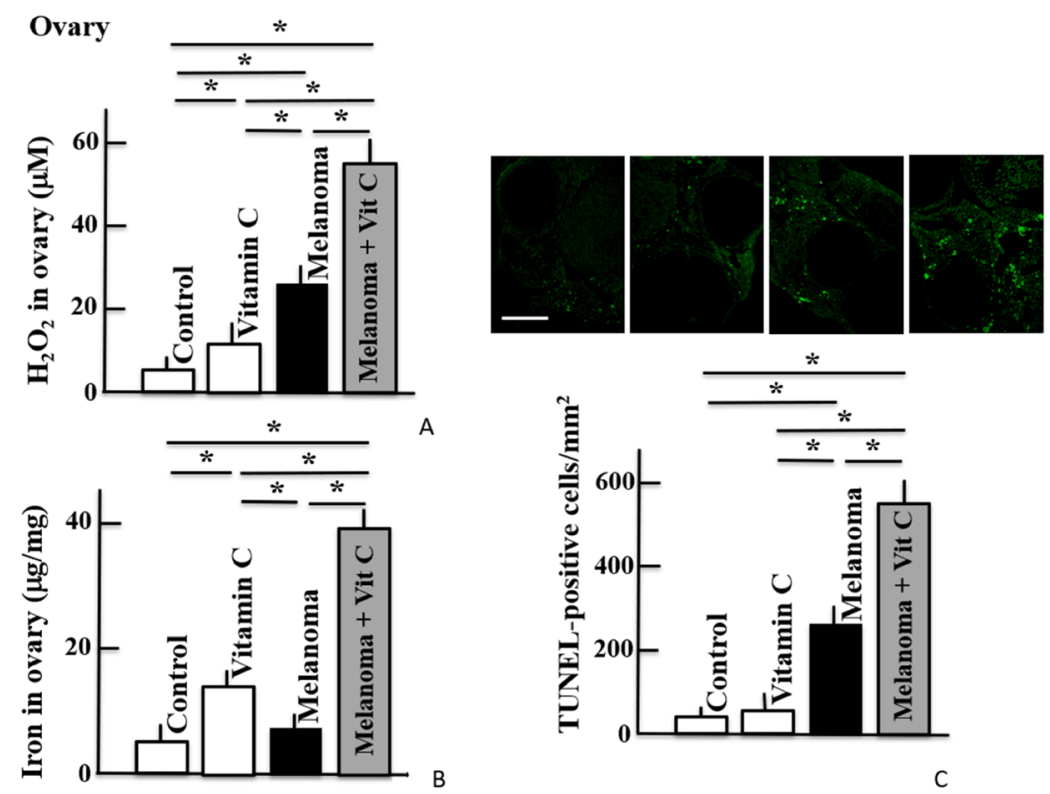

Fig. 6. Effect of High Dose VC Administration on the Concentrations of $\mathrm{H}_{2} \mathrm{O}_{2}$ (A) and Iron (B), and Number of TUNEL-Positive Cells (C) in the Ovary Samples of the Experimental and Control Groups

The data are represented as mean \pm S.D. Tukey's post-hoc test was used to compare the differences between the groups. ${ }^{*} p<0.05$. Scale bar $=100 \mu$ m. Abbreviation: Vit C, vitamin C. (Color figure can be accessed in the online version.) 


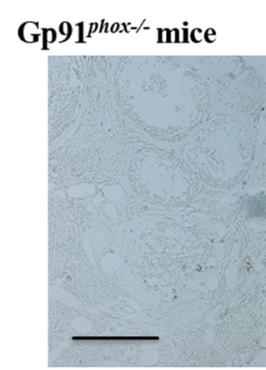

Control

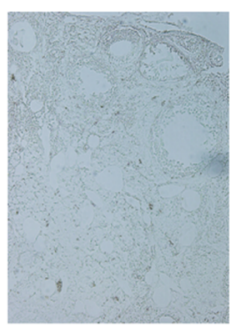

Vitamin C

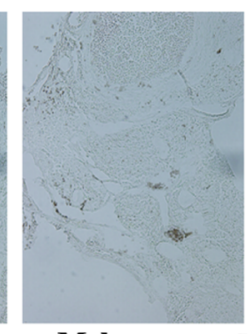

Melanoma Melanoma + Vitamin C

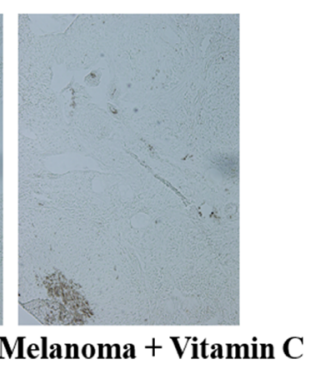

A
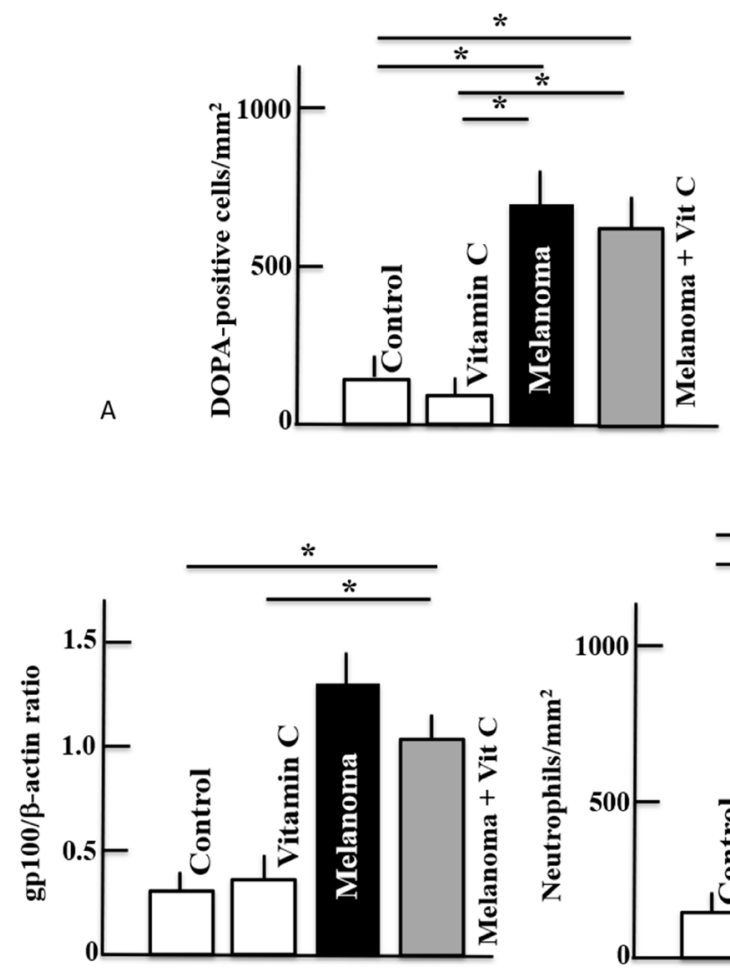

B

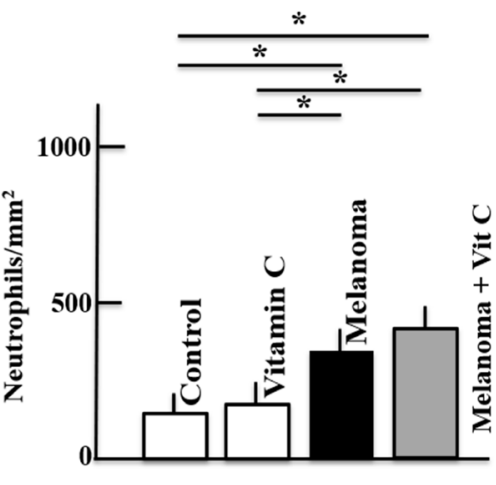

C

Fig. 7. Effect of High Dose VC Administration on the Invasion and Proliferation of Melanoma Cells and the Number of Neutrophils in the Ovaries of gp $91^{\text {phox-l- }}$ Mice

The number of DOPA-positive cells (A), gp100-positive melanoma cells (B), and neutrophils (C) in the experimental and control groups. The data are represented as mean \pm S.D. Tukey's post-hoc test was used to compare the differences between the groups. $* p<0.05$. Scale bar $=100 \mu$ m. Abbreviation: Vit C, vitamin C. (Color figure can be accessed in the online version.)

\section{DISCUSSION}

The results obtained from each organ (lung, liver, jejunum, and ovary) revealed that $\mathrm{VC}$ administration significantly reduced the invasion of melanoma in the ovaries. The number of neutrophils was increased in the $\mathrm{VC}+$ melanoma-administered mice. Moreover, $\mathrm{H}_{2} \mathrm{O}_{2}$ and iron concentrations in the ovaries were significantly increased in the $\mathrm{VC}+$ melanoma-administered mice. However, in the gp $91^{\text {phox-l- }}$ mice, the numbers of DOPA-positive cells, gp100-positive melanoma cells, and neutrophils were not different between the melanoma group and the $\mathrm{VC}+$ melanoma group.

In our previous study, in a model of sodium dextran sulfate (DSS)-induced ulcerative colitis, administration of high-dose VC regulated neutrophil and cell apoptosis and improved ulcerative colitis. ${ }^{23)}$ In addition, irinotecan-induced skin dryness was improved by administering high-dose VC via ROS. ${ }^{24)}$ Furthermore, neutrophils and ROS were involved in azoxymethane + DSS-induced colorectal cancer. ${ }^{19)}$ In this study, neutrophils also play an important role in melanoma, suggesting that neutrophils are crucial in the pathological function of high-dose VC. In this study, the effect of VC on the invasion and proliferation of melanoma cells was found to be organ-specific. In addition, neutrophils in the ovaries were increased upon VC administration. Neutrophils are involved in the ovulation and maintenance of the sexual cycle,${ }^{25)}$ and it is thought that neutrophils rich in $\mathrm{VC}$ accumulate in the ovaries because they take up VC. However, the detailed mechanism is unknown. Nevertheless, when neutrophils infiltrate the ovary, they release $\beta$-endorphins, an analgesic peptides, to maintain the sexual cycle. ${ }^{26,27)} \beta$-Endorphin reportedly acts on the theca and granulosa cells surrounding the follicle to regulate the production of steroid hormones. ${ }^{28,29)}$ Therefore, this may explain how increased neutrophilic infiltration in the ovaries help to maintain the sexual cycle. Moreover, VC has also shown to stimulate the production ${ }^{30-33)}$ and function $^{34,35)}$ of leukocytes, especially neutrophils. In addition to the increase in neutrophil count in the ovaries, MIP-2, which is a neutro- 
phil chemotactic factor, was also elevated by VC administration. Surprisingly, VC did not induce neutrophil production in the other organs examined. Thus, VC can promote cancer cells death by increasing the number of neutrophils and stimulating their phagocytic activity. Interestingly, VC administration in normal mice did not change in leukocyte production or function. This suggests that VC may be more effective when immune cells are damaged; however, further research is warranted.

Neutrophils release ROS to kill cancer cells, neutrophils themselves tend to be damaged. ${ }^{36)}$ However, Neutrophils generally store high concentrations of $\mathrm{VC}^{37)}$ and protect themselves against oxidative damage. ${ }^{38,39)} \mathrm{VC}$ is protected from neutrophil autoxidation by its antioxidant function. ${ }^{40,41)}$ As melanoma of the ovary increases, so does the neutrophils infiltration. In this study, a large concentration of VC was stored in the neutrophils, and it may because a high-dose of VC assisted in defending the autoxidation mechanism.

Furthermore, high VC concentration causes a Fenton reaction with iron around cancer cells to generate $\mathrm{H}_{2} \mathrm{O}_{2}$. ${ }^{42)}$ Normal cells are not affected because they contain catalase which neutralizes $\mathrm{H}_{2} \mathrm{O}_{2}$, while cancer cells are affected because they are catalase-deficient. ${ }^{43}$ In the present study, ovaries of the $\mathrm{VC}+$ melanoma-administered mice showed increased $\mathrm{H}_{2} \mathrm{O}_{2}$ and iron concentrations, and apoptotic cells, suggesting increased melanoma cell death. These results indicate that VC can safely and effectively reduce the levels of melanoma cells in the ovary, and it may be promising as a therapeutic agent for melanoma.

Finally, to validate the effect of neutrophils, we used gp91 $1^{\text {phox-l- }}$ mice. The effect of $\mathrm{VC}$ was attenuated in gp91 $1^{\text {phox-l- }}$ mice, suggesting that $\mathrm{H}_{2} \mathrm{O}_{2}$ generated by gp91 phox (NADPH oxidase 2) expressed in neutrophils caused cancer cell death. Therefore, it is speculated that gp91 phox plays an important role in suppressing cancer cells as well as the action of neutrophils themselves.

\section{CONCLUSION}

High-dose VC showed organ-specific effects and suppressed the invasion and proliferation of melanoma in the ovaries. VC administration increased the neutrophil count in the ovaries compared to other organs, and promoted cancer cell death by stimulating their phagocytic function. Moreover, gp $91^{\text {pho }}$ in neutrophils plays an important role in suppressing cancer cells. Therefore, it can be expected that VC can safely and effectively treat melanoma cells in the ovaries. However, because this study was performed in mice, the results need to be validated using human trials.

Acknowledgments This study was supported by JSPS KAKENHI (Grant No. 18K06082).

Conflict of Interest The authors declare no conflict of interest.

\section{REFERENCES}

1) Saida $T$. The concept of de novo origin of cutaneous malignant melanoma. Eur. J. Dermatol., 4, 252-254 (1994).

2) Baderca F, Vincze D, Balica N, Solovan C. Mucosal melanomas in the elderly: challenging cases and review of the literature. Clin. Interv. Aging, 9, 929-937 (2014).

3) Saida T. Diagnosis and treatment of cutaneous malignant melanoma. Skin Cancer, 8 (Special), 161-186 (1993).

4) Berwick M, Wiggins C. The current epidemiology of cutaneous malignant melanoma. Front. Biosci., 11, 1244-1254 (2006).

5) $\mathrm{Hu} \mathrm{S}$, Ma F, Collado-Mesa F, Kirsner RS. UV radiation, latitude, and melanoma in US Hispanics and blacks. Arch. Dermatol., 140, $819-824$ (2004).

6) Ishihara K, Saida T, Yamamoto A. Updated statistical data for malignant melanoma in Japan. Int. J. Clin. Oncol., 6, 109-116 (2001).

7) Sabban F, Boukerrou M, Mubiayi N, Houpeau JL, Robert Y, Vinatier D. Malignant melanoma metastatic to the ovary. A case report. Gynecol. Obstet. Fertil., 33, 409-411 (2005).

8) Gök ND, Yildiz K, Corakçi A. Primary malignant melanoma of the ovary: case report and review of the literature. Turk. Patoloji. Derg., 27, 169-172 (2011).

9) Chiba $Y$, Ihata $Y$, Nakayama $H$. Two cases of malignant melanoma arising primarily in the female genital organ. Skin Cancer, 21, 368-371 (2006)

10) Tsuchida $T$, Koga $H$, Uhara $H$, Kiyohara $T$, Takenouti $T$, Anzai S, Umebayashi Y, Kadono T, Kamiya H, Kouno T, Shikama N, Tsutsumida A, Nakamura Y, Namikawa K, Hatta N, Murata Y, Moroi Y, Iwatsuki K. Guidelines for the treatment of skin malignant tumors second edition. JPN. J. Dermatol., 125, 5-75 (2015).

11) Ichimura T. Malignant melanoma. Adv. Obstet. Gynecol., 69, 60-62 (2017).

12) Bucher A, White N. Vitamin $\mathrm{C}$ in the prevention and treatment of the common cold. Am. J. Lifestyle Med., 10, 181-183 (2016).

13) Li Y, Schellhorn HE. New developments and novel therapeutic perspectives for vitamin C. J. Nutr., 137, 2171-2184 (2007).

14) Rose RC, Bode AM. Biology of free radical scavengers: an evaluation of ascorbate. FASEB J., 7, 1135-1142 (1993).

15) Monti DA, Mitchell E, Bazzan AJ, Littman S, Zabrecky G, Yeo CJ, Pillai MV, Newberg AB, Deshmukh S, Levine M. Phase I evaluation of intravenous ascorbic acid in combination with gemcitabine and erlotinib in patients with metastatic pancreatic cancer. PLOS ONE, 7, e29794 (2012).

16) Takahashi H, Mizuno H, Yanagisawa A. High-dose intravenous vitamin $\mathrm{C}$ improves quality of life in cancer patients. Pers. Med. Universe, 1, 49-53 (2012).

17) Frei B, Lawson S. Vitamin $\mathrm{C}$ and cancer revisited. Proc. Natl. Acad. Sci. U.S.A., 105, 11037-11038 (2008).

18) Hiramoto K, Tanaka H, Katada T, Konishi H, Hayakawa R. Effect of $17 \beta$-estradiol on ultraviolet radiation-induced immunosuppression and carcinogenesis in mice. Environ. Dermatol., 5, 147-154 (1998).

19) Kondo K, Sano R, Goto K, Hiramoto K, Ooi K. Administration of high-dose vitamin $\mathrm{C}$ and irinotecan ameliorates colorectal cancer induced by azoxymethane and dextran sodium sulfate in mice. Biol. Pharm. Bull., 41, 1797-1803 (2018).

20) Jimbow K, Uesugi Y. New melanogenesis and photobiological processes in activation and proliferation of precursor melanocytes after UV-exposure: ultrastructural differentiation of precursor melanocytes from Langerhans cells. J. Invest. Dermatol., 78, 108-115 (1982).

21) Yokoyama S, Hiramoto K, Koyama M, Ooi K. Skin disruption is associated with indomethacin-induced small intestinal injury in mice. Exp. Dermatol., 23, 659-663 (2014).

22) Hiramoto K, Sugiyama D, Takahashi $Y$, Mafune E. The amelioration effect of tranexamic acid in wrinkles induced by skin dryness. Biomed. Pharmacother., 80, 16-22 (2016).

23) Kondo K, Hiramoto K, Yamate Y, Goto K, Sekijima H, Ooi K. Ameliorative effect of high-dose vitamin $\mathrm{C}$ administration on dextran sulfate sodium-induced colitis mouse model. Biol. Pharm. Bull., 42, 954-959 (2019).

24) Nakanishi K, Goto K, Kondo K, Hiramoto K, Ooi K. Irinotecan-in- 
duced skin dryness is ameliorated by orally administered high-dose vitamin C in mice. J. Exp. Pharmacol., 11, 109-114 (2019).

25) Sonoda Y, Mukaida N, Wang JB, Shimada-Hiratsuka M, Naito M, Kasahara T, Harada A, Inoue M, Matsushima K. Physiologic regulation of postovulatory neutrophil migration into vagina in mice by a C-X-C chemokine(s). J. Immunol., 160, 6159-6165 (1998).

26) Sasaki S, Tamaki Y, Nagata K, Kobayashi Y. Regulation of the estrous cycle by neutrophils via opioid peptides. J. Immunol., 187, 774-780 (2011)

27) Machelska H, Stein C. Leukocyte-derived opioid peptides and inhibition of pain. J. Neuroimmune Pharmacol., 1, 90-97 (2006).

28) Kaminski T, Siawrys G, Bogacka I, Okrasa S, Przala J. The regulation of steroidogenesis by opioid peptides in porcine theca cells. Anim. Reprod. Sci., 78, 71-84 (2003).

29) Kaminski T, Siawrys C, Bogacka I, Okrasa S, Przala J. The influence of opioid peptides on steroidogenesis in porcine granulosa cells. Reprod. Domest. Anim., 39, 25-32 (2004).

30) Prinz W, Bortz R, Bregin B, Hersch M. The effect of ascorbic acid supplementation on some parameters of the human immunological defence system. Int. J. Vitam. Nutr. Res., 47, 248-257 (1977).

31) Vallance $S$. Relationships between ascorbic acid and serum proteins of the immune system. BMJ, 2, 437-438 (1977).

32) Kennes B, Dumont I, Brohee D, Hubert C, Neve P. Effect of vitamin C supplements on cell-mediated immunity in old people. Gerontology, 29, 305-310 (1983).

33) Panush RS, Delafuente JC, Katz P, Johnson J. Modulation of certain immunologic responses by vitamin C. III. Potentiation of in vitro and in vivo lymphocyte responses. Int. J. Vitam. Nutr. Res. Suppl., 23, 35-47 (1982).

34) Anderson R, Oosthuizen R, Maritz R, Theron A, Van Rensburg AJ.
The effects of increasing weekly doses of ascorbate on certain cellular and humoral immune functions in normal volunteers. $\mathrm{Am} . J$. Clin. Nutr., 33, 71-76 (1980).

35) Levy R, Shriker O, Porath A, Riesenberg K, Schlaeffer F. Vitamin $\mathrm{C}$ for the treatment of recurrent furunculosis in patients with impaired neutrophil functions. J. Infect. Dis., 173, 1502-1505 (1996).

36) Alberts B, Lewis J, Raff M, Roberts K, Watson JD. Differentiated cells and the maintenance of tissues. Molecular biology of the cell. (3rd ed.), Garland Publishing, Inc., New York, pp. 1139-1193 (1994).

37) Washko PW, Wang Y, Levine M. Ascorbic acid recycling in human neutrophils. J. Biol. Chem., 268, 15531-15535 (1993).

38) Bergsten P, Amitai G, Kehrl J, Dhariwal KR, Klein HG, Levine M. Millimolar concentrations of ascorbic acid in purified human mononuclear leukocytes. Depletion and reaccumulation. J. Biol. Chem., 265, 2584-2587 (1990).

39) Washko P, Rotrosen D, Levine M. Ascorbic acid transport and accumulation in human neutrophils. J. Biol. Chem., 264, 18996-19002 (1989).

40) Wolf G. Uptake of ascorbic acid by human neutrophils. Nutr. Rev., 51, 337-338 (1993).

41) Jarwalla RJ, Harakeh S. Mechanisms underlying the action of vitamin $\mathrm{C}$ in viral and immunodeficiency disease. Vitamin $C$ in health and disease. (Lester $\mathrm{P}$, Jurgen $\mathrm{F}$ eds.), Marcel Dekker, Inc., New York, pp. 309-322 (1997).

42) Mojić M, Pristov BJ, Maksimović-Ivanić D, Jones DR, Stanić M, Mijatović S, Spasojević I. Extracellular iron diminishes anticancer effects of vitamin C: an in vitro study. Sci. Rep., 4, 5955 (2014).

43) Frei B, Lawson S. Vitamin C and cancer revisited. Proc. Natl. Acad. Sci. U.S.A., 105, 11037-11038 (2008). 